\title{
Chemokine Expression in Human Astrocytes in Response to Shiga Toxin 2
}

\author{
Naomi Kioka, Koichi Minami, Akira Tamura, and Norishige Yoshikawa \\ Department of Pediatrics, Wakayama Medical University School of Medicine, 811-1 Kimiidera, Wakayama 641-0012, Japan \\ Correspondence should be addressed to Naomi Kioka, knaomiharu@yahoo.co.jp
}

Received 13 September 2012; Revised 7 November 2012; Accepted 7 November 2012

Academic Editor: Andrew S. Day

Copyright () 2012 Naomi Kioka et al. This is an open access article distributed under the Creative Commons Attribution License, which permits unrestricted use, distribution, and reproduction in any medium, provided the original work is properly cited.

Infection with Shiga toxin- (Stx-) producing Escherichia coli can lead to hemolytic uremic syndrome (HUS). Approximately, 30\% of patients with HUS suffer from complications in the central nervous system (CNS), which is an important determinant of mortality in such patients. Autopsy shows mostly edema and hypoxic-ischemic changes in the CNS, often with microhemorrhages. It has been suggested that Stx-induced damage to human brain endothelial cells, which are essential constituents of the blood-brain barrier, plays a crucial role in the development of the CNS complications. However, it is unclear whether Stx affects brain neuroglial cells. In the present study, we investigated the direct involvement of Stx in the inflammatory responses of human astrocytes (HASTs) treated with Stx. Immunohistochemistry and real-time PCR revealed that the expression of globotriaosylceramide (Gb3), the receptor for Stx2, and Gb3 synthase (GalT6) in HASTs was increased by interleukin-1 $\beta$ (IL-1 $\beta$ ). Expression of both interleukin8 (IL-8) and monocyte chemoattractant protein-1 (MCP-1) mRNA in HASTs was significantly upregulated by Stx2. These results suggest that Stx2 induces inflammatory responses, particularly through expression of chemokines, in HASTs expressing Gb3 and may, thus, affect brain glial cells, playing a key role in the pathogenesis of CNS manifestations associated with HUS.

\section{Introduction}

Hemolytic uremic syndrome (HUS) is the most frequent cause of acute renal failure in children. The traditional diagnostic criteria for this syndrome include hemolytic anemia with fragmented erythrocytes, thrombocytopenia, and renal failure [1]. The epidemic form of HUS occurs mostly as a result of hemorrhagic colitis. In $90 \%$ of HUS cases, infection with Shiga toxin- (Stx-) producing Escherichia coli (STEC) is strongly implicated [2].

Stx is divided into two main groups, referred to as Stx 1 and Stx2, which share approximately 56\% amino acid sequence similarity [3]. Stx1 is essentially identical to the Stx produced by Shigella dysenteriae. Stx 2 is a more potent cause of severe HUS and central nervous system (CNS) impairment. Stx is a 71-kDa protein composed of a $32-k D a A$ subunit (StxA) and five 7.7-kDa B subunits (StxB). StxA has $\mathrm{N}$-glycosidase activity that removes the adenine of the $28 \mathrm{~S}$ RNA within the $60 \mathrm{~S}$ ribosomal subunit, thereby rendering ribosomes inactive for protein synthesis [4]. StxB is essential for transport of StxA to the cytosol. StxB specifically binds to the cell membrane of the globotriaosylceramide (Gb3) receptor, which is present in specific mammalian host cells [5]. Stx is then internalized with Gb3 and transported toward the trans-Golgi network [6].

Stx is thought to enter the bloodstream through inflamed intestinal mucosa, then bind with low affinity to blood cells, specifically polymorphonuclear leukocytes, thereby being transported to the target organs [7]. Gb3, the functional receptor for Stx, is present on the surfaces of various cell types, including human glomerular endothelial cells, glomerular epithelial cells [8], and human brain endothelial cells (HBECs) [9]. Stx binding to target cells expressing Gb3 inhibits protein synthesis and induces apoptosis and necrosis [10].

Approximately, 30\% of patients with HUS suffer from CNS complications, and these patients have the poorest prognosis [11]. However, the pathogenesis of CNS dysfunction is not fully understood. Although thrombotic microangiopathic damage is characteristic of HUS and is thought to result from the direct cytotoxic effects of circulating Stx on the vascular endothelium [12], there is no consensus on the 
pathogenic mechanisms responsible for brain involvement in HUS. However, a possible role for the inflammatory response provoked by cytokines has been suggested. Clinical studies have demonstrated that patients with HUS have elevated plasma levels of cytokines such as tumor necrosis factor $\alpha$ (TNF- $\alpha$ ), interleukin-1 $\beta$ (IL-1 $\beta$ ), and interleukin-6 (IL-6) [13]. In the presence of TNF- $\alpha$ or IL- $1 \beta$, the cytotoxicity of Stx towards endothelial cells is significantly increased. Furthermore, TNF- $\alpha$ and IL- $1 \beta$ upregulate the expression of Gb3 in endothelial cells [14]. Similarly, although it has been demonstrated that HBECs are very resistant to Stxinduced cytotoxicity [9], inflammatory mediators, such as TNF- $\alpha$ and/or IL- $1 \beta$, markedly increase the sensitivity of HBECs to Stx cytotoxicity [15], which is due to modulation of expression of $\mathrm{Gb} 3$.

Several components of the Gb3 metabolic pathway may be involved in cytokine-stimulated Gb3 expression. Gb3 is synthesized from lactosylceramide and UDP-galactose by Gb3 synthase (GalT6) [16]. Consequently, increased Gb3 content could be due to increased activity of GalT6. Eisenhauer et al. have shown that TNF- $\alpha$ and IL- $1 \beta$ increase GalT6 activity and mRNA levels [17].

The inflammation associated with HUS is marked by the release of chemokines, and the levels of IL- 8 and MCP- 1 are significantly increased in urine samples collected from HUS patients [18]. Purified Stxs directly induce the expression of the neutrophil chemoattractant IL- 8 in human intestinal epithelial cells [19]. These Stx-induced increases in IL-8 synthesis by intestinal epithelial cells could be important in augmenting the host mucosal inflammatory responses to STEC infection [20]. Endothelial cells exposed to Stx2 release inflammatory chemokines, such as IL-8 and MCP-1, which stimulate adhesion and transmigration of leukocytes [21]. Such chemokines may also play a role in the pathogenesis of HUS. However, in brain parenchymal cells and neuroglial cells, expression of chemokines in response to Stx has not been studied.

Several studies support the hypothesis that Stx causes brain injury in HUS. Intravenous inoculation of rabbits with Stx2 caused severe CNS injury associated with the invasion of Stx2 through the BBB [22]. Some authors have reported that intracerebroventricular administration of Stx2 causes neuronal death and glial cell damage in the rat brain. Transmission electron microscopy studies have revealed apoptotic neurons, ultrastructural alterations of glia, and demyelinated fibers [23]. In addition, confocal microscopy has demonstrated reactive astrocytes in contact with Stx2-containing neurons [24]. These findings support the contention that Stx2 has a direct effect on brain neuroglial cells.

The brain contains neuronal, glial (e.g., astrocytes, microglia, and oligodendrocytes), and endothelial cells. Human astrocytes (HASTs) are the most abundant type of glial cell in the human brain. They play key roles in development, homeostasis, inflammatory responses, and repair of the CNS by producing a wide variety of cytokines, chemokines, and growth factors and by expressing receptors for these molecules [25]. These chemokines play important roles in development, growth, cell migration, production of free radicals, apoptosis, T-cell activation, neoplasia, inflammatory regulation in response to injury, wound healing, tissue repair, and macrophage recruitment, as well as in interactions with pathogens, including viruses [26-29]. Chemokines and their receptors play important roles in several neurodegenerative and inflammatory diseases of the CNS, including trauma, stroke, Alzheimer's disease, multiple sclerosis, CNS tumors, and human immunodeficiency virus encephalitis [30-32]. We speculated that Stxs would act directly on astrocytes by inducing the production of chemokines and play a role in CNS complications.

In the present study, we investigated how Gb3 expression, including GalT6, in HASTs is controlled by inflammatory cytokines. In addition, we sought to obtain further evidence that Stx2 acts to produce chemokines in HASTs and that these chemokines participate in the pathogenesis of CNS complications associated with HUS.

\section{Methods}

2.1. Reagents and Toxins. Purified Stx 2 was purchased from Toxin Technology Inc. (Sarasota, FL, USA). Toxicity was determined to be greater than $10^{7}$ units per milligram using a Vero cell assay. All reagents were purchased from Sigma Chemical Co. (St. Louis, MO, USA).

2.2. Cells and Culture Conditions. HASTs derived from human fetal brain tissue (Lonza, Walkersville, MD, USA) were routinely subcultured every 6 days in Clonetics Astrocyte Growth Medium (Lonza). Cells were reseeded after reaching confluence at 3,500 cells per square centimeter and were incubated at $37^{\circ} \mathrm{C}$ in a humidified atmosphere containing $5 \% \mathrm{CO}_{2}$. The HASTs in the experiments were used after two to four passages.

2.3. Analysis of Gb3 Expression Stimulated by $I L-1 \beta$. Analysis of Gb3 expression stimulated by IL- $1 \beta$ was conducted by immunohistochemistry using the Vectastain Elite ABC kit (Vector Labs, Burlingame, CA, USA). HASTs were cultured with $10^{-6} \mathrm{~mol} / \mathrm{L}$ IL- $1 \beta$ or with medium alone. The cells were stimulated for $48 \mathrm{~h}$ with IL- $1 \beta$ and fixed in $4 \%$ paraformaldehyde for $10 \mathrm{~min}$ at room temperature. Slides were prepared and incubated in rat anti-human CD77 IgM antibody ( 1 : 100 dilution; Beckman Coulter, Brea, CA, USA) or PBS for $8 \mathrm{~h}$ at room temperature, then washed and incubated with a 1:100 dilution of secondary antibody (supplied in the Vectastain kit) at room temperature for $1 \mathrm{~h}$. The colorimetric reaction was obtained using $0.5 \mathrm{mg} / \mathrm{mL}$ diaminobenzidine (DAB) substrate solution (Vector Labs) with $0.02 \% \mathrm{H}_{2} \mathrm{O}_{2}$. The slides were counterstained with Gill's hematoxylin.

2.4. Real-Time Quantitative PCR of GalT-6 mRNA. HASTs grown in six-well plates were incubated in IL- $1 \beta\left(10^{-8}\right.$ to $\left.10^{-5} \mathrm{~mol} / \mathrm{L}\right)$ or TNF- $\alpha\left(10^{-8}\right.$ to $\left.10^{-5} \mathrm{~mol} / \mathrm{L}\right)$. At 0,8 , and $24 \mathrm{~h}$ postinoculation, total RNA was isolated using the RNeasy Mini kit (Qiagen, Valencia, CA, USA), with an RNase-free DNase (Qiagen) treatment for $30 \mathrm{~min}$ in accordance with the 
manufacturer's instructions. RNA was reverse transcribed to produce cDNA using the Prime Script RT reagent kit (Takara Bio, Otsu, Japan). Real-time quantitative polymerase chain reaction (PCR) was performed using SYBR Green PCR Master Mix and the Thermal Cycler Dice Real-Time System (Takara Bio). All experiments were carried out five times. The real-time PCR primers specific for GalT6 and the housekeeping gene glyceraldehyde-3-phosphate dehydrogenase (GAPDH) are listed in Table 1. The PCR conditions were $95^{\circ} \mathrm{C}$ for $10 \mathrm{~s}$, followed by 40 cycles of $95^{\circ} \mathrm{C}$ for $5 \mathrm{~s}$ and $60^{\circ} \mathrm{C}$ for $30 \mathrm{~s}$. The relative amounts of mRNA were determined from the threshold cycle values of the reference gene, GAPDH. All further calculations and statistical analyses were carried out based on these values and are referred to as relative expression ratios.

2.5. Chemokine Enzyme-Linked Immunosorbent Assay (ELI$S A)$. HASTs grown in six-well plates were incubated in $10^{-9}$ and $10^{-10} \mathrm{~mol} / \mathrm{L}$ Stx 2 . At 0,24 , and $48 \mathrm{~h}$ postinoculation, cell supernatants from treated HASTs were collected by centrifugation and stored at $-80^{\circ} \mathrm{C}$ until further analysis. The ELISAs for IL-8 and monocyte chemoattractant protein-1 (MCP-1) were performed using the corresponding Quantikine Colorimetric Sandwich ELISA kits (R\&D Systems, Minneapolis, $\mathrm{MN}, \mathrm{USA}$ ) in accordance with the manufacturer's protocol. Chemokines were quantified by measurement of absorbance at $450 \mathrm{~nm}\left(A_{450}\right)$ with a 680 Microtiter Plate Reader (BioRad Laboratories, Hercules, CA, USA). Chemokine protein concentrations were calculated based on the standard curves. The assay sensitivities were $4 \mathrm{pg} / \mathrm{mL}$ (IL-8) and $5 \mathrm{pg} / \mathrm{mL}$ (MCP-1).

2.6. Real-Time Quantitative PCR of Chemokine mRNA. HASTs grown in six-well plates were incubated with either $10^{-9}$ or $10^{-10} \mathrm{~mol} / \mathrm{L} \mathrm{Stx} 2,10^{-9} \mathrm{~mol} / \mathrm{L} \mathrm{Stx} 2$ with $10^{-6} \mathrm{~mol} / \mathrm{L}$ IL- $1 \beta$, or $10^{-6} \mathrm{~mol} / \mathrm{L}$ IL- $1 \beta$ alone. At 0,8 , and $24 \mathrm{~h}$ postinoculation, total RNA was extracted and real-time quantitative PCR performed using the methods described previously. The real-time PCR primers specific for IL- 8 and MCP-1 (Takara Bio) are listed in Table 1. The PCR conditions were one cycle at $95^{\circ} \mathrm{C}$ for $10 \mathrm{~s}$, followed by 40 cycles of $95^{\circ} \mathrm{C}$ for $5 \mathrm{~s}$ and $60^{\circ} \mathrm{C}$ for $30 \mathrm{~s}$. The relative amounts of the mRNA were determined as described previously.

2.7. Statistics. The means $\pm \mathrm{SD}$ were determined, and the mean values in the two separate groups were compared using a Student's $t$-test. Differences at $P<0.05$ were considered to be significant.

\section{Results}

3.1. Immunohistochemical Analysis of Gb3 Expression in HASTs Treated with $I L-1 \beta$. Proinflammatory cytokines increase the expression of Stx receptor Gb3 on the surface of different Stx target cells. To determine the levels of Gb3 expression in HASTs treated with IL- $1 \beta$, immunohistochemistry was performed. In HASTs treated for $48 \mathrm{~h}$ with $10^{-6} \mathrm{~mol} / \mathrm{L}$ IL- $1 \beta$, a relatively high level of Gb3
(Figure 1(a)) was detected in comparison to the untreated controls (Figure 1(b)). Conversely, no change in Gb3 level was detected in HASTs treated with TNF- $\alpha$ (data were not shown).

3.2. Real-Time Quantitative PCR Analysis of GalT6 mRNA Induction in HASTs Treated with IL-1 3 or TNF- $\alpha$. It has been demonstrated using HBECs and intestinal epithelial cells that inflammatory cytokines increase Gb3 expression levels by generating lactosylceramide through the activation of GalT6. Immunohistochemistry revealed that the expression of Gb3 in HASTs was increased by IL- $1 \beta$. In addition, we investigated the expression of GalT6 mRNA relative to that of the untreated controls following treatment with IL- $1 \beta$ $\left(10^{-8}\right.$ to $\left.10^{-5} \mathrm{~mol} / \mathrm{L}\right)$ or TNF- $\alpha\left(10^{-8}\right.$ to $\left.10^{-5} \mathrm{~mol} / \mathrm{L}\right)$ for 8 or $24 \mathrm{~h}$. At $8 \mathrm{~h}$, IL- $1 \beta\left(10^{-7}\right.$ to $\left.10^{-5} \mathrm{~mol} / \mathrm{L}\right)$ treatment resulted in significant induction $(P<0.05)$ of GalT6 mRNA relative to the untreated controls (Figure $2(\mathrm{a})$ ). A 6.5 -fold increase in the level of GalT6 mRNA was induced with $10^{-6} \mathrm{~mol} / \mathrm{L}$ IL- $1 \beta$. TNF- $\alpha$ treatment showed a similar trend to IL- $1 \beta$ treatment at $8 \mathrm{~h}$, but it was not significant (Figure 2(b)).

\subsection{Expression of Chemokine mRNA in HASTs upon Stim-} ulation with Stx2. Astrocytes are an important source of chemokines that play a role in brain inflammatory responses. To investigate whether expression of IL- 8 and MCP- 1 mRNA was increased in HASTs treated with Stx2, we used realtime PCR. Following treatment with $10^{-10}$ to $10^{-9} \mathrm{~mol} / \mathrm{L}$ Stx 2 for 8 or $24 \mathrm{~h}$, real-time quantitative PCR was performed to verify the expression of IL- 8 and MCP-1 transcripts. Following treatment with Stx2, IL-8 mRNA induction was significantly elevated $(P<0.05)$ relative to the untreated controls (Figure 3(a)). A 256-fold increase in the level of IL$8 \mathrm{mRNA}$ was induced by $10^{-10} \mathrm{~mol} / \mathrm{L} \mathrm{Stx} 2$, and a 1,155 -fold increase was induced with $10^{-9} \mathrm{~mol} / \mathrm{L}$ Stx 2 at $8 \mathrm{~h}$. A 5.7 fold increase of MCP-1 mRNA was induced by $10^{-10} \mathrm{~mol} / \mathrm{L}$ Stx2, and an 11.6-fold increase was induced with $10^{-9} \mathrm{~mol} / \mathrm{L}$ Stx 2 at $8 \mathrm{~h}$, in comparison with the untreated controls (Figure 3(b)).

3.4. Chemokine Release by HASTs upon Stimulation with Stx2. To determine whether the upregulation of chemokine mRNA was accompanied by release of these chemokines into the medium, HASTs were exposed to $10^{-9} \mathrm{~mol} / \mathrm{L}$ and $10^{-10} \mathrm{~mol} / \mathrm{L} \mathrm{Stx} 2$ for 24 and $48 \mathrm{~h}$, and the concentrations of chemokines in the medium were measured by ELISA. Treatment of HASTs with $10^{-9} \mathrm{~mol} / \mathrm{L}$ and $10^{-10} \mathrm{~mol} / \mathrm{L} \mathrm{Stx} 2$ increased secretion of IL- 8 into the medium relative to the control cultures at 24 and $48 \mathrm{~h}$ (Figure $4(\mathrm{a})$ ). The concentrations of IL- 8 in the medium were significantly higher in the Stx2-treated cultures, especially for $10^{-9} \mathrm{~mol} / \mathrm{L} \mathrm{Stx} 2$, than those in the control cultures at 24 and $48 \mathrm{~h}$. Similarly, treatment of HASTs with $10^{-9} \mathrm{~mol} / \mathrm{L}$ and $10^{-10} \mathrm{~mol} / \mathrm{L} \mathrm{Stx} 2$ increased the secretion of MCP- 1 into the medium relative to the control cultures at 24 and $48 \mathrm{~h}$ (Figure $4(\mathrm{~b})$ ). Unlike IL-8, MCP-1 was released in the absence of Stx 2 stimulation (Figure 4(b)). 
TABle 1: Primer sequences.

\begin{tabular}{lll}
\hline Gene & Primer $($ forward $)$ & Primer $($ reverse $)$ \\
\hline GalT6 & ACCTGCGGAACCTGACCAAC & CATGCACAGCGCCATGAAC \\
IL-8 & ACACTGCGCCAACACAGAAATTA & TTTGCTTGAAGTTTCACTGGCATC \\
MCP-1 & GCTCATAGCAGCCACCTTCATTC & GGACACTTGCTGCTGGTGATTC \\
GAPDH & GCACCGTCAAGGCTGAGAAC & TGGTGAAGACGCAGTGGA \\
\hline
\end{tabular}

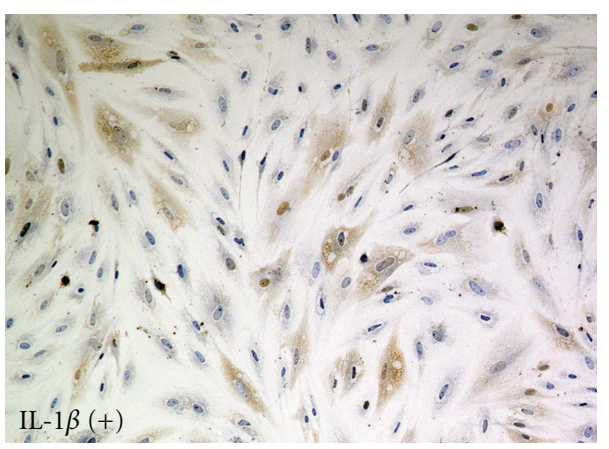

(a)

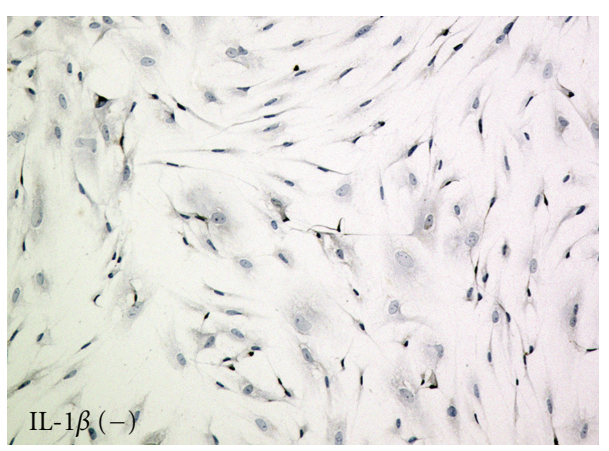

(b)

FIGURE 1: Immunohistochemical analysis of Gb3 expression in HASTs. Immunohistochemistry was performed on HASTs treated with IL- $1 \beta$. A condensed brown DAB precipitate was observed in HASTs. A relatively high level of Gb3 was detected in HASTs treated with $10^{-6}$ mol/L IL- $1 \beta$ for $48 \mathrm{~h}$ (a), compared with the negative control (b) (original magnification $\times 100$ ).

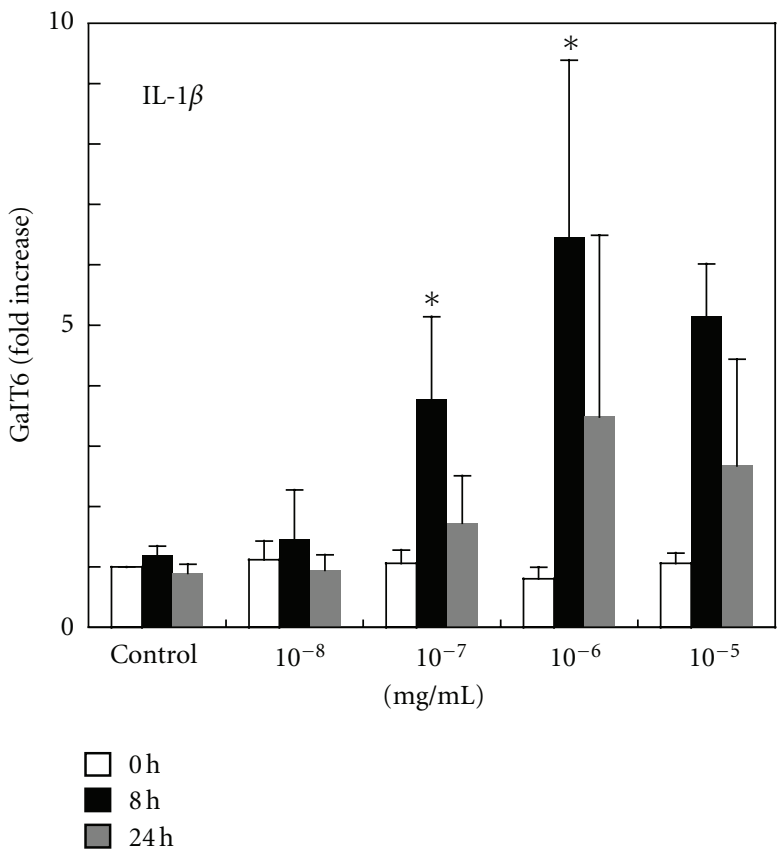

(a)

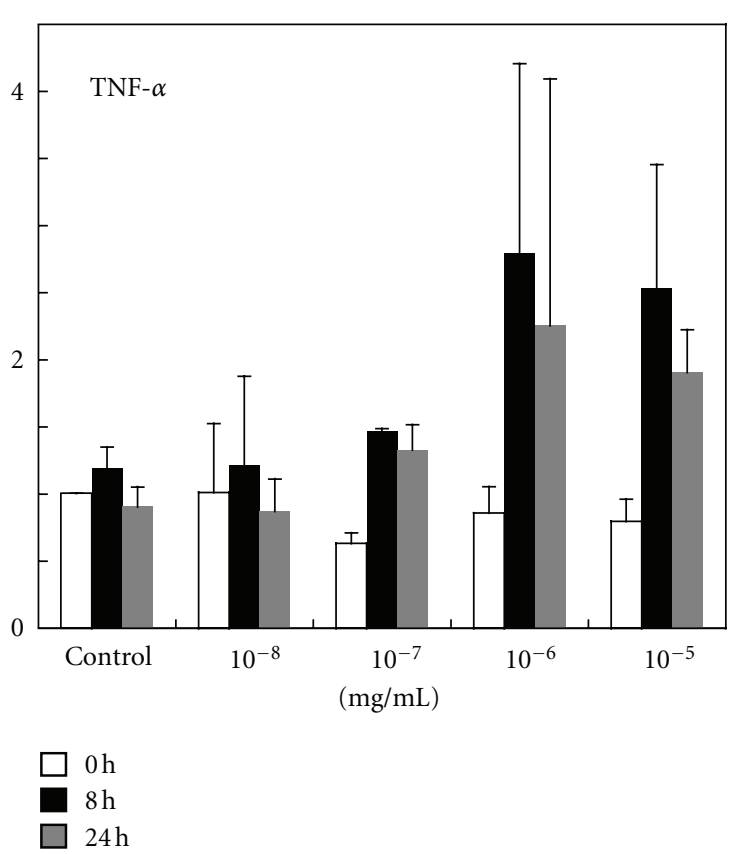

(b)

FIGURe 2: Real-time quantitative PCR analysis of GalT6 mRNA induction in HASTs treated with IL-1 $\beta$ and TNF- $\alpha$. Expression of GalT6 mRNA was examined by real-time quantitative PCR using HASTs treated with IL- $1 \beta\left(10^{-8}\right.$ to $\left.10^{-5} \mathrm{~mol} / \mathrm{L}\right)$ (a) and TNF- $\alpha$ ( $10^{-8}$ to $10^{-5} \mathrm{~mol} / \mathrm{L}$ ) (b) for 8 or $24 \mathrm{~h}$. The levels of induction of GalT6 mRNA expression were calculated relative to the control cultures. Data are expressed as the fold increase of mRNA (mean \pm SD; SD values from five independent experiments). Comparison of GalT6 mRNA levels elicited by treatment with IL- $1 \beta$ or TNF- $\alpha$ with those of the untreated control. ${ }^{*} P<0.05$. 


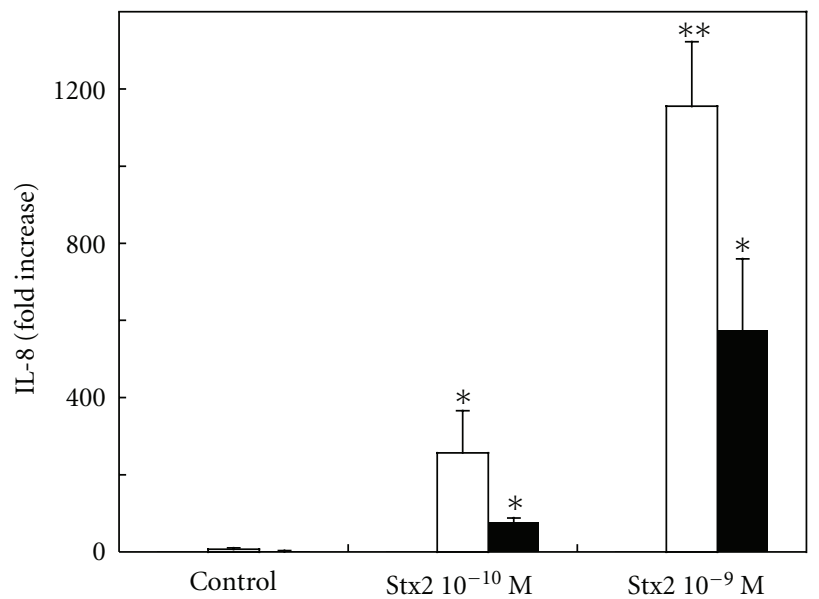

$\square 0 \mathrm{~h}$

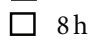

$24 \mathrm{~h}$

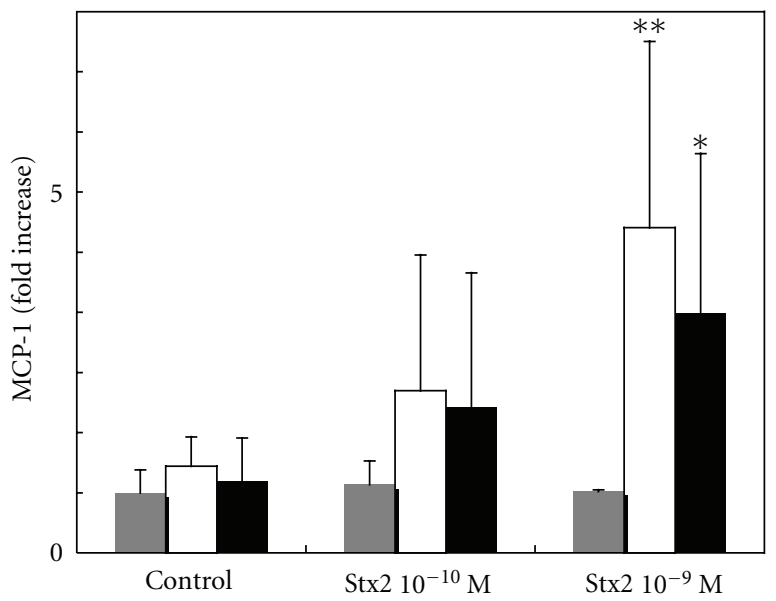

$\square \quad 0 \mathrm{~h}$
$\square \quad 8 \mathrm{~h}$
$\square 24 \mathrm{~h}$

(a)

(b)

FIgURE 3: Expression of chemokine mRNA in HASTs upon stimulation with Stx2. The expressions of IL-8 (a) and MCP-1 (b) mRNA was examined by real-time quantitative PCR using HASTs treated with $10^{-10}$ to $10^{-9} \mathrm{~mol} / \mathrm{L}$ Stx 2 for 8 or 24 h. The levels of induction of IL8 and MCP-1 mRNA were calculated relative to the control cultures. Data are expressed as fold increases of mRNA (mean \pm SD from five independent experiments). Comparisons were made between the levels of IL- 8 and MCP-1 mRNA elicited by Stx 2 and those of the untreated controls. ${ }^{*} P<0.05$ and ${ }^{* *} P<0.01$.
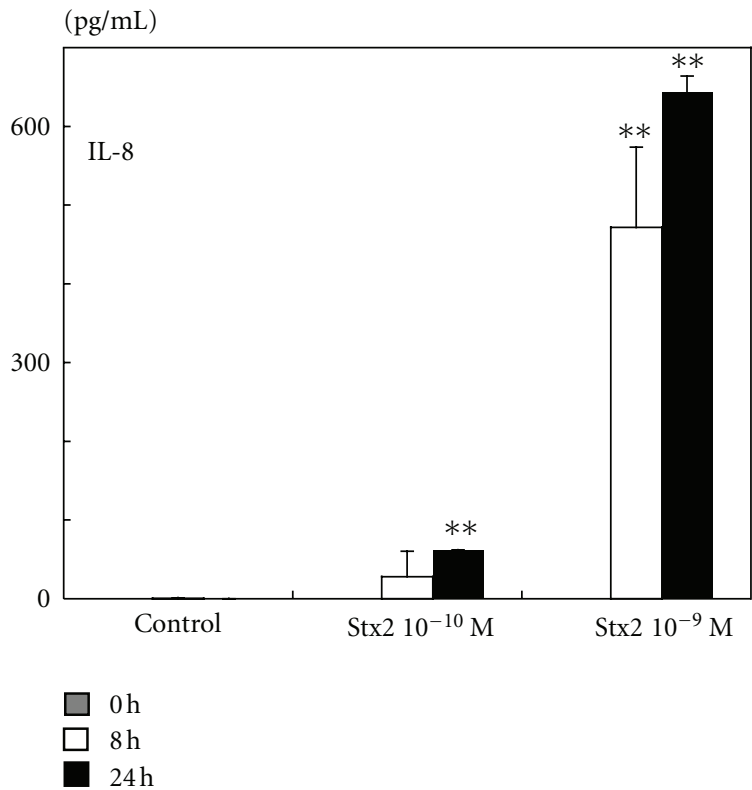
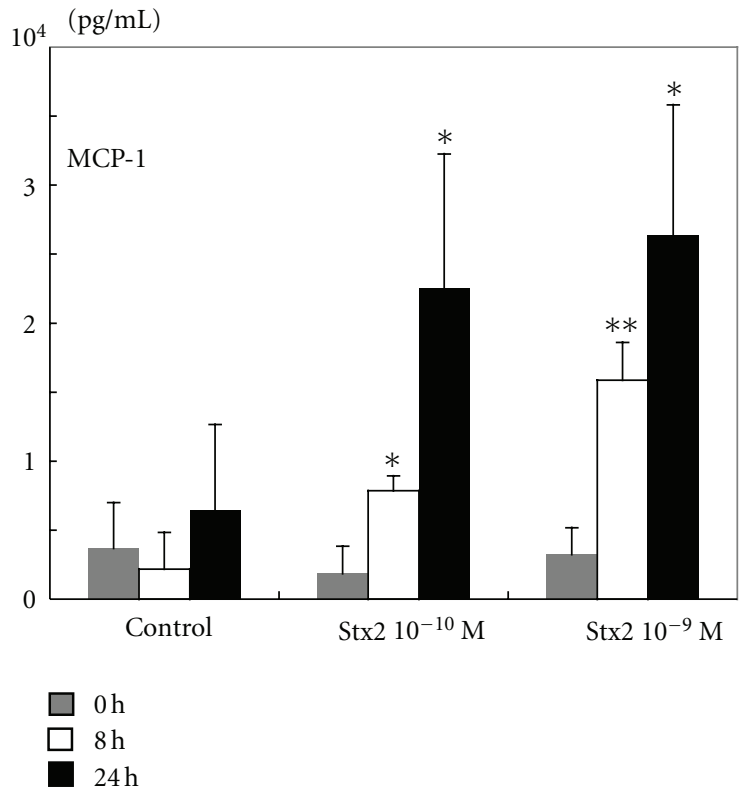

(a)

(b)

FIgure 4: Chemokine release by HASTs upon stimulation with Stx2. Secretion of IL-8 (a) and MCP-1 (b) by HASTs treated with $10^{-9}$ or $10^{-10} \mathrm{~mol} / \mathrm{L}$ Stx 2 for 24 or $48 \mathrm{~h}$. IL- 8 and MCP- 1 were measured in cell culture supernatants using ELISA. Data are the means \pm SD from the five experiments. Levels of Stx2-induced IL- 8 or MCP-1 and those in the untreated controls were calculated. ${ }^{*} P<0.05$ and ${ }^{* *} P<0.01$. 


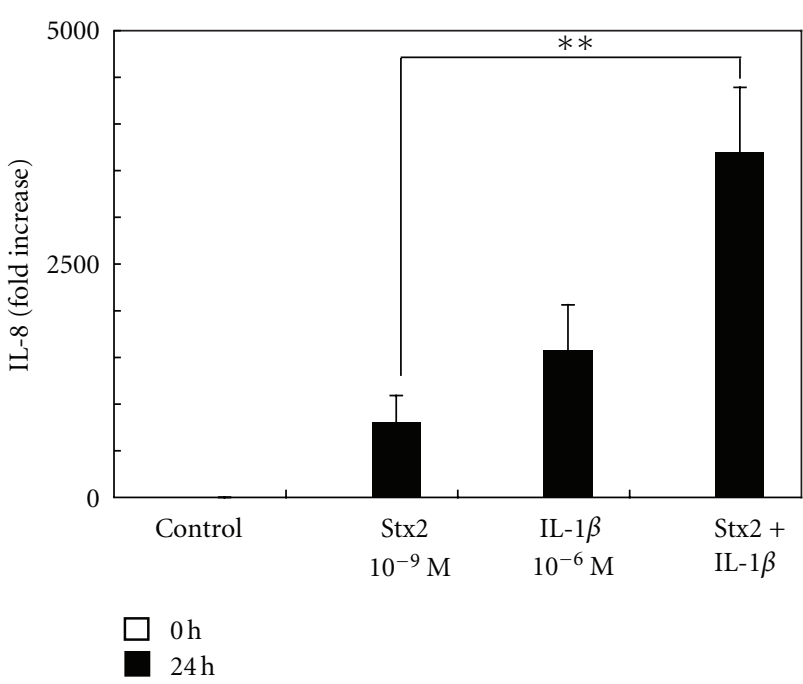

FIgURe 5: Expression of IL-8 mRNA in HASTs upon stimulation with Stx 2 and IL-1 $\beta$. Expression of IL- 8 mRNA was examined by real-time quantitative PCR using HASTs treated with $10^{-9} \mathrm{~mol} / \mathrm{L}$ Stx 2 and $10^{-6} \mathrm{~mol} / \mathrm{L}$ IL- $1 \beta$ for $24 \mathrm{~h}$. The levels of induction of IL8 mRNA expression were calculated relative to those of the control cultures, which had not been treated with Stx 2 or IL-1 $\beta$. Data are expressed as fold increases of mRNA expression (mean \pm SD from five independent experiments). Comparisons between the levels of IL- 8 mRNA elicited by treatment with $10^{-9} \mathrm{~mol} / \mathrm{L}$ Stx 2 alone and those elicited with $10^{-9} \mathrm{~mol} / \mathrm{L}$ Stx 2 and $10^{-6} \mathrm{~mol} / \mathrm{L} \mathrm{IL}-1 \beta$ are shown. Induction of IL- 8 mRNA upon stimulation with $10^{-9} \mathrm{~mol} / \mathrm{L}$ Stx 2 and $10^{-6} \mathrm{~mol} / \mathrm{L}$ IL- $1 \beta$ in combination at $24 \mathrm{~h}$ was significantly elevated $(P<0.01)$ in comparison with that elicited by either $10^{-9} \mathrm{~mol} / \mathrm{L}$ Stx 2 or $10^{-6} \mathrm{~mol} / \mathrm{L}$ IL- $1 \beta$ alone. ${ }^{* *} P<0.01$.

\subsection{Expression of IL-8 mRNA in HASTs upon Stimulation} with Stx 2 and $I L-1 \beta$. Using real-time PCR, we found that the expression of GalT6 mRNA was increased by stimulation with IL- $1 \beta$, and immunohistochemistry demonstrated that the level of Gb3 expression in HASTs was also increased by IL- $1 \beta$ stimulation. To investigate whether the increase of Gb3 expression induced by IL- $1 \beta$ stimulation correlated with the expression of chemokine mRNA, we treated HASTs with Stx2 and IL- $1 \beta$. Induction of IL- 8 mRNA upon stimulation with $10^{-9} \mathrm{~mol} / \mathrm{L}$ Stx 2 and $10^{-6} \mathrm{~mol} / \mathrm{L} \mathrm{IL}-1 \beta$ combined was significantly greater $(P<0.05)$ at $24 \mathrm{~h}$ than that resulting from stimulation with either agent alone at the same concentration (Figure 5). A 3,700-fold increase was induced by this combination of Stx 2 and IL-1 $\beta$, in comparison with the control levels. Coincubation of Stx 2 with IL- $1 \beta$ did not elicit a significant increase in the level of MCP-1 mRNA (data were not shown).

\section{Discussion}

CNS dysfunction is an important determinant of prognosis and mortality in children with HUS [11]. Although the pathogenesis of CNS involvement in HUS is not fully understood, the disruption and increased permeability of the $\mathrm{BBB}$ and neuronal disturbance resulting from HBEC injury are central events in CNS complications observed during the acute phase of HUS [15].

In a previous histopathologic study, Mizuguchi et al. showed that intravenous administration of Stx 2 to rabbits resulted in hemorrhage and necrosis of both neural and endothelial cells in the brain [33]. Moreover, an in vivo study has shown that Stx2 injected intravenously into rabbits was detected in the cerebrospinal fluid (CSF) and that influx of Stx2 into the CSF caused injury to the tight junctions between ependymal cells, resulting in destruction of the BBB [16]. Some authors have reported that intracerebroventricular administration of Stx 2 causes neuronal death and glial cell damage in the rat brain [23]. However, it is unclear how Stx2 affects neuroglial cells during an inflammatory response.

HASTs comprise $55 \%-60 \%$ of the total number of human brain cells [34] and are involved in virtually every type of brain pathology. HASTs play key roles in the development, inflammation, and repair of the CNS by producing a wide variety of chemokines. Under inflammatory or otherwise pathologic conditions, HASTs become activated and display enhanced production of several cytokines, chemokines, and growth factors. In addition, HASTs generally start to proliferate under such conditions, a phenomenon known as astrogliosis. Therefore, knowledge of the cellular response of HASTs to Stx is essential to understand the neuropathology observed in the severe cases of HUS.

In this study, IL- $1 \beta$ treatment of HASTs increased both Gb3 and GalT6 mRNA expression, as determined by immunohistochemistry and real-time PCR, respectively. In addition, treatment of HASTs with Stx 2 induced production of the chemokines IL-8 and MCP-1. A mixture of Stx2 and IL- $1 \beta$ induced IL- 8 to a marked degree. These findings suggest that Stx 2 triggers an immune response through the expression of chemokines in HASTs.

Toxin binding to Gb3 is the primary determinant of the cytotoxic and pathologic effects of Stx protein. Previous studies have highlighted the role of proinflammatory agents in enhancing the sensitivity of different target cells, including HBECs, to the effects of Stx through upregulation of Gb3 expression [9, 35]. Gb3-expressing cells are recognized to be the targets of Stx. Primary HBECs become susceptible to Stx when they express Gb3 in response to TNF- $\alpha$, further exacerbating the disease process [15]. Therefore, we investigated whether Gb3 expression in HASTs is increased by inflammatory cytokines. Using real-time PCR, we determined that the expression of GalT6 mRNA was increased by IL- $1 \beta$ stimulation, and immunohistochemistry showed that the level of Gb3 expression in HASTs was increased by IL- $1 \beta$ stimulation. These results demonstrated that HASTs, which are CNS parenchymal cells, express Gb3, and that this expression is increased by inflammatory cytokines. In HBECs, IL- $1 \beta$ and TNF- $\alpha$ increased the expression of Gb3 and enhanced the toxicity of Stx. Only IL- $1 \beta$ increased the expression of GalT6 mRNA and increased the level of Gb3 expression in HASTs. Treatment with TNF- $\alpha$ elicited a response similar to that for IL- $1 \beta$ in terms of the expression of GalT6 mRNA at $8 \mathrm{~h}$, although the degree of similarity was not significant. In this study, the mechanism by which IL- $1 \beta$ increased the level of Gb3 expression in HASTs more 
than TNF- $\alpha$ is not understood, although it may be a result of differences in cell types, and further investigations of this are required.

Chemokines, a family of proinflammatory cytokines, are divided into two subfamilies: the CXC family, represented in this study by IL-8, and the CC family, represented here by MCP-1. Chemokines stimulate target cell-specific directional migration of leukocytes and may be potent mediators of inflammatory processes. Human IL- 8 is a proinflammatory chemokine secreted predominantly by monocytes, endothelial cells, and glial cells and acts as a chemoattractant and activator of neutrophils via CXCR1 and CXCR2 receptor signaling. MCP-1 is a potent chemotactic factor for monocytes and plays an important role in the regulation of repair processes and cellular interactions in the CNS $[25,26]$. It can be expressed by many cell types including microglia, astrocytes, and neurons [20,21].

In recent years, it has been shown that chemokines play many roles in inflammation in the CNS and are responsible for the development of various neurodegenerative diseases and ischemic damage to brain cells $[17,18]$. MCP-1 and IL8 are upregulated in the brains of patients with Alzheimer's disease [24]. In addition, the levels of MCP-1 and IL-8 are significantly elevated in the CSF of infants with amyotrophic lateral sclerosis, Japanese encephalitis, or hypoxia-ischemia [36].

We found that by stimulating HASTs with Stx2 chemokines such as IL- 8 and MCP- 1 were produced, the production of IL- 8 being particularly marked. Elevated levels of IL- 8 and MCP-1 mRNA were found in toxin-treated cells in comparison to the untreated controls. IL- 8 and MCP-1 proteins were found at higher levels in the conditioned media of Stx2-treated cells than in those of untreated cells, and the chemokines IL- 8 and MCP-1 were produced by HASTs upon stimulation with Stx. Several studies have demonstrated that Stx induces the expression of IL- 8 and MCP-1 in intestinal epithelial cells, endothelial cells, and monocytes and that the levels of IL- 8 and MCP-1 are significantly increased in urine samples collected from patients with HUS. It has, therefore, been suggested that chemokines such as IL-8 and MCP-1 play a specific role in the pathogenesis of HUS. For HUS patients, a high peripheral polymorphonuclear neutrophil count at presentation was strongly associated with a poor prognosis [37]. Activated polymorphonuclear neutrophils could contribute to the brain inflammatory response and participate in the secondary damage following BBB disruption in HUS. Stx 2 induces expression of several chemokines, such as IL-8 and MCP-1, in astrocytes, and might constitutively induce the inflammatory response. In a mouse model of HUS, neutralization of chemokines resulted in decreased renal fibrin deposition, suggesting that disrupting or eliminating these macrophage chemokines may be a useful treatment for the kidney damage associated with HUS [38]. Currently, it is unclear whether IL-8 and MCP-1 are induced in the CNS. In the present study, we found that astrocytes were responsive to Stx 2 and produced IL-8 and MCP-1. In biological systems, further studies will need to address whether IL- 8 and MCP- 1 are induced by Stx 2 in the CNS, and what effects they exert.
No therapy for HUS encephalopathy has yet been established. However, Fujii et al. showed that betamethasone sodium phosphate pulse therapy reduced the mortality rate and prolonged the survival period of rabbits with Stx2toxemia [39]. Betamethasone sodium phosphate is routinely used as a steroid therapy for patients with brain edema. Dexamethasone reduces the permeability of the normal $\mathrm{BBB}$ in mice [40]. In brain astrocytes, Kim et al. showed the coordinated regulation of angiotensin-1 and vascular endothelial growth factor (VEGF) by dexamethasone, suggesting a novel mechanism underlying glucocorticoidinduced stabilization of the BBB [41]. In addition, Rock et al. examined cytokine and chemokine expression with and without dexamethasone in human microglia and astrocytes infected with Mycobacterium tuberculosis. They reported that treatment with dexamethasone effectively suppressed production of these mediators [42]. Steroid protective effects are thought to be mediated by immunomodulatory activities, such as a decrease in the production of proinflammatory cytokines. Therefore, anti-inflammatory compounds may have therapeutic potential for Stx-induced neurological manifestation. Investigations into the glucocorticoid regulation of chemokine expression by Stx 2 in HASTs are currently underway.

In summary, our results suggest a possible relationship between excessive chemokine production in the CNS and brain damage once Stx 2 reaches the brain parenchyma. We propose that suppression of chemokine production could be a new strategy for treatment of CNS damage in HUS encephalopathy.

\section{Conclusions}

In this study, we investigated the inflammatory responses in human astrocytes treated with Stx2. Immunohistochemistry and real-time PCR revealed that the expression of globotriaosylceramide (Gb3), the receptor for Stx2, and Gb3 synthase (GalT6) in HASTs was increased by interleukin-1 $\beta$ (IL-1 $\beta$ ). Chemokine mRNA expression in astrocytes was significantly upregulated by Stx 2 . Chemokines in the CNS may be associated with the pathogenesis of CNS manifestations with HUS.

\section{Acknowledgments}

The authors would like to thank Dr. Masakazu Miyawaki for the technical advice and Dr. Koichi Nakanishi for reviewing the paper.

\section{References}

[1] B. S. Kaplan, T. G. Cleary, and T. G. Obrig, "Recent advances in understanding the pathogenesis of the hemolytic uremic syndromes," Pediatric Nephrology, vol. 4, no. 3, pp. 276-283, 1990.

[2] M. A. Karmali, M. Petric, and C. Lim, "The association between idiopathic hemolytic uremic syndrome and infection by verotoxin-producing Escherichia coli," Journal of Infectious Diseases, vol. 151, no. 5, pp. 775-782, 1985. 
[3] A. D. O'Brien and R. K. Holmes, "Shiga and Shiga-like toxins," Microbiological Reviews, vol. 51, no. 2, pp. 206-220, 1987.

[4] T. G. Obrig, T. P. Moran, and J. E. Brown, "The mode of action of Shiga toxin on peptide elongation of eukaryotic protein synthesis," Biochemical Journal, vol. 244, no. 2, pp. 287-294, 1987.

[5] C. A. Lingwood, "Role of verotoxin receptors in pathogenesis," Trends in Microbiology, vol. 4, no. 4, pp. 147-153, 1996.

[6] F. Mallard, C. Antony, D. Tenza, J. Salamero, B. Goud, and L. Johannes, "Direct pathway from early/recycling endosomes to the Golgi apparatus revealed through the study of Shiga toxin B-fragment transport," Journal of Cell Biology, vol. 143, no. 4, pp. 973-990, 1998.

[7] D. M. Te Loo, L. A. H. Monnens, T. J. Van Der Velden et al., "Binding and transfer of verocytotoxin by polymorphonuclear leukocytes in hemolytic uremic syndrome," Blood, vol. 95, no. 11, pp. 3396-3402, 2000.

[8] A. K. Hughes, D. I. Schmid, and D. E. Kohan, "Sex steroids do not affect shigatoxin cytotoxicity on human renal tubular or glomerular cells," BMC Nephrology, vol. 3, article no. 1, 7 pages, 2002.

[9] P. B. Eisenhauer, P. Chaturvedi, R. E. Fine et al., "Tumor necrosis factor alpha increases human cerebral endothelial cell Gb3 and sensitivity to Shiga toxin," Infection and Immunity, vol. 69, no. 3, pp. 1889-1894, 2001.

[10] T. Taguchi, H. Uchida, N. Kiyokawa et al., "Verotoxins induce apoptosis in human renal tubular epithelium derived cells," Kidney International, vol. 53, no. 6, pp. 1681-1688, 1998.

[11] K. J. Sheth, H. M. Swick, and N. Haworth, "Neurological involvement in hemolytic-uremic syndrome," Annals of Neurology, vol. 19, no. 1, pp. 90-93, 1986.

[12] V. L. Tesh, J. E. Samuel, L. P. Perera, J. B. Sharefkin, and A. D. O'Brien, "Evaluation of the role of Shiga and Shiga-like toxins in mediating direct damage to human vascular endothelial cells," Journal of Infectious Diseases, vol. 164, no. 2, pp. 344 $352,1991$.

[13] N. C. A. J. Van de Kar, R. W. Sauerwein, P. N. M. Demacker, G. E. Grau, V. W. M. Van Hinsbergh, and L. A. H. Monnens, "Plasma cytokine levels in hemolytic uremic syndrome," Nephron, vol. 71, no. 3, pp. 309-313, 1995.

[14] P. A. Van Setten, V. W. M. Van Hinsbergh, T. J. A. N. Van Der Velden et al., "Effects of TNF $\alpha$ on verocytotoxin cytotoxicity in purified human glomerular microvascular endothelial cells," Kidney International, vol. 51, no. 4, pp. 1245-1256, 1997.

[15] P. K. Stricklett, A. K. Hughes, Z. Ergonul, and D. E. Kohan, "Molecular basis for up-regulation by inflammatory cytokines of Shiga toxin 1 cytotoxicity and globotriaosylceramide expression," Journal of Infectious Diseases, vol. 186, no. 7, pp. 976-982, 2002.

[16] Y. Kojima, S. Fukumoto, K. Furukawa et al., "Molecular cloning of globotriaosylceramide/CD77 synthase, a glycosyltransferase that initiates the synthesis of globo series glycosphingolipids," Journal of Biological Chemistry, vol. 275, no. 20, pp. 15152-15156, 2000.

[17] P. B. Eisenhauer, M. S. Jacewicz, K. J. Conn et al., "Escherichia coli Shiga toxin 1 and TNF- $\alpha$ induce cytokine release by human cerebral microvascular endothelial cells," Microbial Pathogenesis, vol. 36, no. 4, pp. 189-196, 2004.

[18] P. A. Van Setten, V. W. Van Hinsbergh, L. P. Van Den Heuvel et al., "Monocyte chemoattractant protein-1 andinterleukin- 8 levels in urine and serum of patients with hemolytic uremic syndrome," Pediatric Research, vol. 43, no. 6, pp. 759-767, 1998.

[19] C. Yamasaki, Y. Natori, X. T. Zeng et al., "Induction of cytokines in a human colon epithelial cell line by Shiga toxin 1 (Stx1) and Stx2 but not by non-toxic mutant Stx1 which lacks N-glycosidase activity," FEBS Letters, vol. 442, no. 2-3, pp. 231-234, 1999.

[20] C. M. Thorpe, B. P. Hurley, L. L. Lincicome, M. S. Jacewicz, G. T. Keusch, and D. W. K. Acheson, "Shiga toxins stimulate secretion of interleukin-8 from intestinal epithelial cells," Infection and Immunity, vol. 67, no. 11, pp. 5985-5993, 1999.

[21] L. M. Harrison, C. Van Den Hoogen, W. C. E. Van Haaften, and V. L. Tesh, "Chemokine expression in the monocytic cell line THP-1 in response to purified Shiga toxin 1 and/or lipopolysaccharides," Infection and Immunity, vol. 73, no. 1, pp. 403-412, 2005.

[22] J. Fujii, Y. Kinoshita, T. Kita et al., "Magnetic resonance imaging and histopathological study of brain lesions in rabbits given intravenous verotoxin 2," Infection and Immunity, vol. 64, no. 12, pp. 5053-5060, 1996.

[23] J. Goldstein, C. F. Loidl, V. P. Creydt, J. Boccoli, and C. Ibarra, "Intracerebroventricular administration of Shiga toxin type 2 induces striatal neuronal death and glial alterations: an ultrastructural study," Brain Research, vol. 1161, no. 1, pp. 106-115, 2007.

[24] J. Boccolia, C. F. Loidl, J. J. Lopez-Costa, V. P. Creydt, C. Ibarra, and J. Goldstein, "Intracerebroventricular administration of Shiga toxin type 2 altered the expression levels of neuronal nitric oxide synthase and glial fibrillary acidic protein in rat brains," Brain Research, vol. 1230, no. C, pp. 320-333, 2008.

[25] S. Meeuwsen, C. Persoon-Deen, M. Bsibsi, R. Ravid, and J. M. Van Noort, "Cytokine, chemokine and growth factor gene profiling of cultured human astrocytes after exposure to proinflammatory stimuli," GLIA, vol. 43, no. 3, pp. 243-253, 2003.

[26] K. B. Bacon and J. K. Harrison, "Chemokines and their receptors in neurobiology: perspectives in physiology and homeostasis," Journal of Neuroimmunology, vol. 104, no. 1, pp. 92-97, 2000.

[27] E. Engelhardt, A. Toksoy, M. Goebeler, S. Debus, E. B. Bröcker, and R. Gillitzer, "Chemokines IL-8, GRO $\alpha$, MCP-1, IP-10, and mig are sequentially and differentially expressed during phasespecific infiltration of leukocyte subsets in human wound healing," American Journal of Pathology, vol. 153, no. 6, pp. 1849-1860, 1998.

[28] A. R. Glabinski and R. M. Ransohoff, "Chemokines and chemokine receptors in CNS pathology," Journal of NeuroVirology, vol. 5, no. 1, pp. 3-12, 1999.

[29] J. Hesselgesser and R. Horuk, "Chemokine and chemokine receptor expression in the central nervous system," Journal of Neurovirology, vol. 5, no. 1, pp. 13-26, 1999.

[30] C. McManus, J. W. Berman, F. M. Brett, H. Staunton, M. Farrell, and C. F. Brosnan, "MCP-1, MCP-2 and MCP-3 expression in multiple sclerosis lesions: an immunohistochemical and in situ hybridization study," Journal of Neuroimmunology, vol. 86, no. 1, pp. 20-29, 1998.

[31] R. M. Ransohoff, T. A. Hamilton, M. Tani et al., "Astrocyte expression of mRNA encoding cytokines IP-10 and JE/MCP1 in experimental autoimmune encephalomyelitis," FASEB Journal, vol. 7, no. 6, pp. 592-600, 1993.

[32] V. G. Sasseville, M. M. Smith, C. R. Mackay et al., "Chemokine expression in simian immunodeficiency virus-induced AIDS 
encephalitis," American Journal of Pathology, vol. 149, no. 5, pp. 1459-1467, 1996.

[33] M. Mizuguchi, S. Tanaka, I. Fujii et al., "Neuronal and vascular pathology produced by verocytotoxin 2 in the rabbit central nervous system," Acta Neuropathologica, vol. 91, no. 3, pp. 254-262, 1996.

[34] L. F. Eng and R. S. Ghirnikar, "GFAP and astrogliosis," Brain Pathology, vol. 4, no. 3, pp. 229-237, 1994.

[35] N. C. Van de Kar, L. A. H. Monnens, M. A. Karmali, and V. W. M. Van Hinsbergh, "Tumor necrosis factor and interleukin1 induce expression of the verocytotoxin receptor globotriaosylceramide on human endothelial cells: implications for the pathogenesis of the hemolytic uremic syndrome," Blood, vol. 80, no. 11, pp. 2755-2764, 1992.

[36] M. Minami, T. Katayama, and M. Satoh, "Brain cytokines and chemokines: roles in ischemic injury and pain," Journal of Pharmacological Sciences, vol. 100, no. 5, pp. 461-470, 2006.

[37] V. I. Landoni, M. De Campos-Nebel, P. Schierloh et al., "Shiga toxin 1-induced inflammatory response in lipopolysaccharide-sensitized astrocytes is mediated by endogenous tumor necrosis factor alpha," Infection and Immunity, vol. 78, no. 3, pp. 1193-1201, 2010.

[38] T. R. Keepers, L. K. Gross, and T. G. Obrig, "Monocyte chemoattractant protein 1, macrophage inflammatory protein $1 \alpha$, and RANTES recruit macrophages to the kidney in a mouse model of hemolytic-uremic syndrome," Infection and Immunity, vol. 75, no. 3, pp. 1229-1236, 2007.

[39] J. Fujii, Y. Kinoshita, A. Matsukawa, S. Y. Villanueva, T. Yutsudo, and S. Yoshida, "Successful steroid pulse therapy for brain lesion caused by Shiga toxin 2 in rabbits," Microbial Pathogenesis, vol. 46, no. 4, pp. 179-184, 2009.

[40] E. T. Hedley-Whyte and D. W. Hsu, "Effect of dexamethasone on blood-brain barrier in the normal mouse," Annals of Neurology, vol. 19, no. 4, pp. 373-377, 1986.

[41] H. Kim, J. M. Lee, J. S. Park et al., "Dexamethasone coordinately regulates angiopoietin-1 and VEGF: a mechanism of glucocorticoid-induced stabilization of blood-brain barrier," Biochemical and Biophysical Research Communications, vol. 372, no. 1, pp. 243-248, 2008.

[42] R. B. Rock, S. Hu, G. Gekker et al., "Mycobacterium tuberculosis-induced cytokine and chemokine expression by human microglia and astrocytes: effects of dexamethasone," Journal of Infectious Diseases, vol. 192, no. 12, pp. 2054-2058, 2005. 


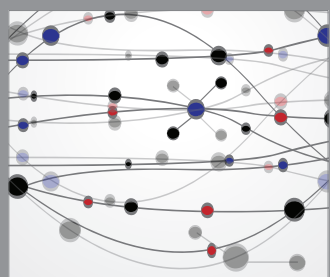

The Scientific World Journal
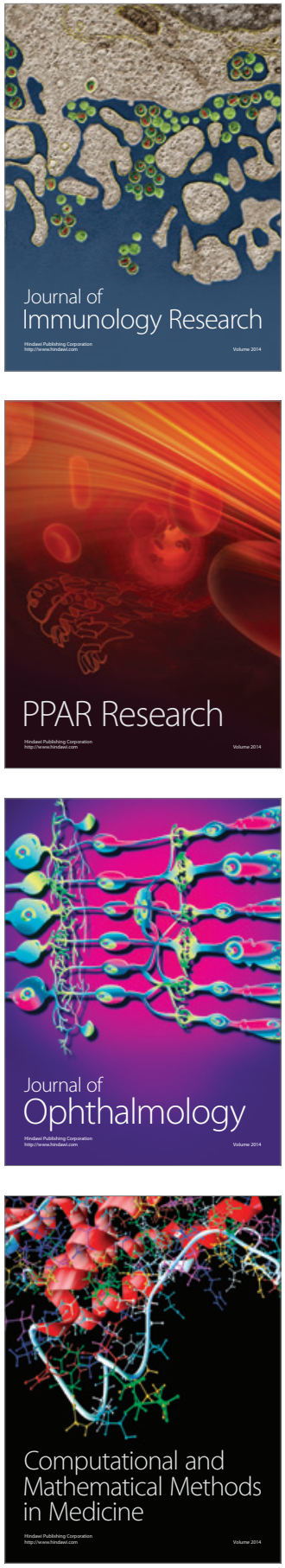

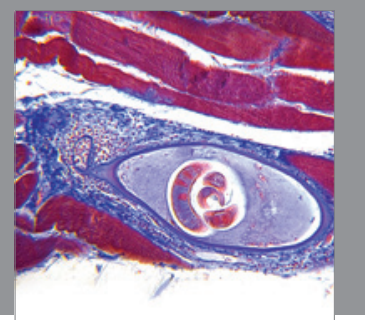

Gastroenterology

Research and Practice
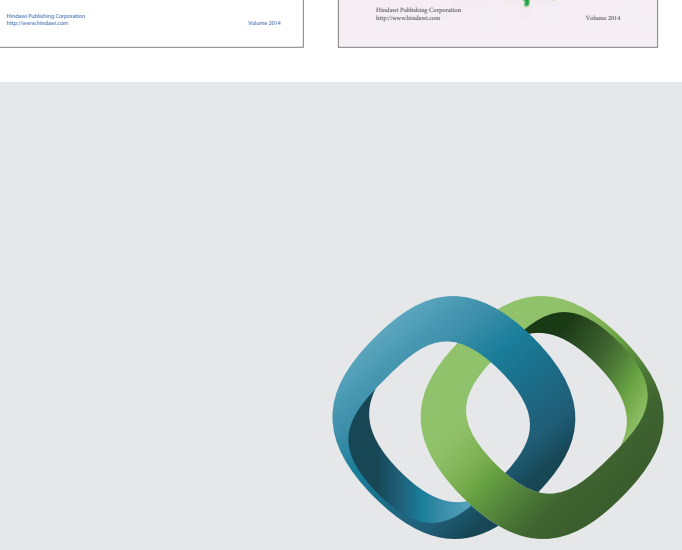

\section{Hindawi}

Submit your manuscripts at

http://www.hindawi.com
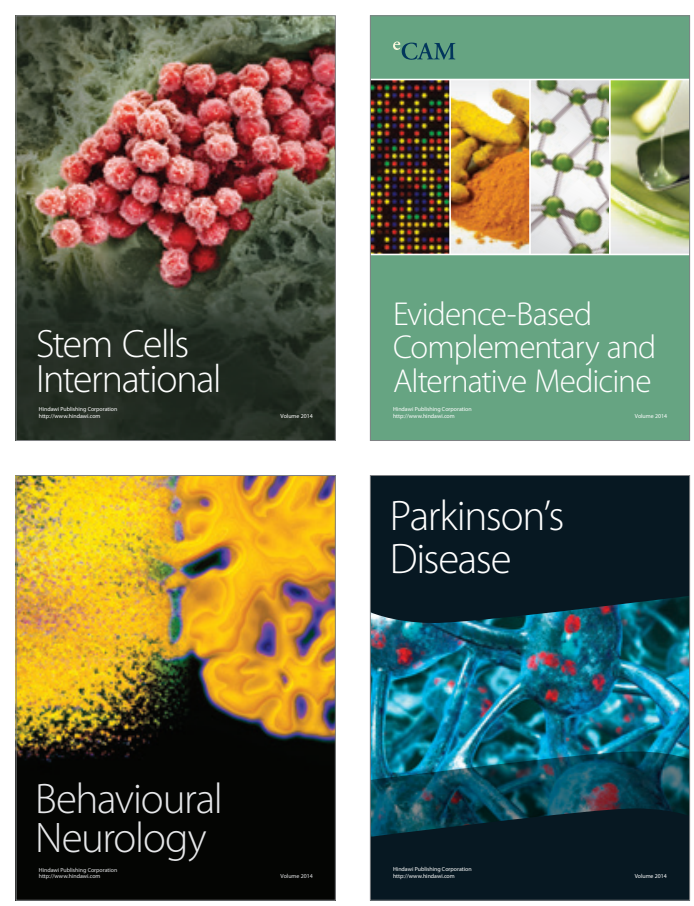

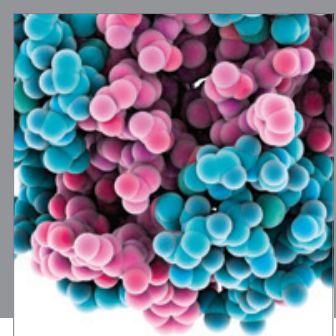

Journal of
Diabetes Research

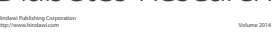

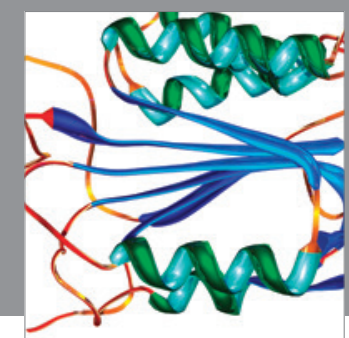

Disease Markers
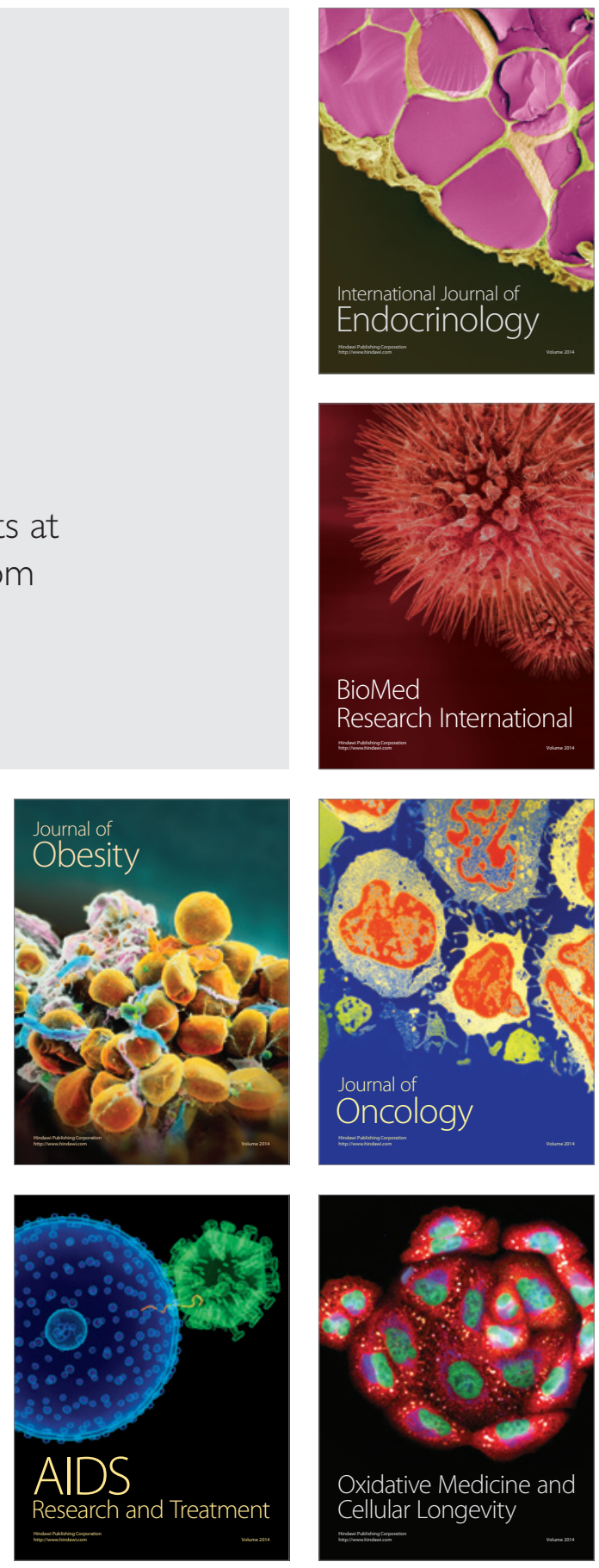